PROCEEDINGS OF THE

AMERICAN MATHEMATICAL SOCIETY

Volume 128, Number 9, Pages 2543-2552

S 0002-9939(99)05408-8

Article electronically published on December 7, 1999

\title{
MIXED MEANS AND INEQUALITIES OF HARDY AND LEVIN-COCHRAN-LEE TYPE FOR MULTIDIMENSIONAL BALLS
}

\author{
ALEKSANDRA ČIŽMEŠIJA, JOSIP PEČARIĆ, AND IVAN PERIĆ \\ (Communicated by Christopher D. Sogge)
}

\begin{abstract}
Integral means of arbitrary order, with power weights and their companion means, where the integrals are taken over balls in $\mathbf{R}^{n}$ centered at the origin, are introduced and related mixed-means inequalities are derived. These relations are then used in obtaining Hardy and Levin-Cochran-Lee inequalities and their companion results for $n$-dimensional balls. Finally, the best possible constants for these inequalities are obtained.
\end{abstract}

\section{INTRODUCTION}

A well-known classical result of G. Hardy (cf. [5], or [8]) states the following:

Theorem 1. Let $p>1$ and $k \neq 1$. Suppose $f$ is a non-negative function such that $x^{1-\frac{k}{p}} f \in L^{p}(0, \infty)$, and the function $F$ is defined on $\langle 0, \infty\rangle$ by

$$
F(x)= \begin{cases}\int_{0}^{x} f(t) d t, & k>1, \\ \int_{x}^{\infty} f(t) d t, & k<1 .\end{cases}
$$

Then

$$
\int_{0}^{\infty} x^{-k} F^{p}(x) d x<\left(\frac{p}{|k-1|}\right)^{p} \int_{0}^{\infty} x^{-k}[x f(x)]^{p} d x,
$$

unless $f \equiv 0$. The constant $\left(\frac{p}{|k-1|}\right)^{p}$ is the best possible.

The famous Hardy's integral inequality (1) is generalized in many different ways by various authors. One possibility of generalizing it is to give its different multivariable analogues. In this paper a generalization of (10) to $n$-dimensional balls will be considered, i.e. the integrals in (1) will be taken over balls in $\mathbf{R}^{n}$ centered at the origin.

Before presenting our idea, let us introduce some notation. If $R>0$ is a real number, let $B(R)=B(\mathbf{0}, R)$ be the ball in $\mathbf{R}^{n}$ centered at the origin and of radius $R$. Further, let $S^{n-1}$ denote the unit sphere in $\mathbf{R}^{n}$, and let $\left|S^{n-1}\right|$ be its area. The volume of the ball $B(R),|B(R)|$, is then $|B(R)|=\int_{B(R)} d \mathbf{x}=\int_{|\mathbf{x}|<R} d \mathbf{x}=$

Received by the editors September 28, 1998.

2000 Mathematics Subject Classification. Primary 26D10, 26D15.

Key words and phrases. Mixed means, Hardy inequality, Levin-Cochran-Lee inequality.

(C)2000 American Mathematical Society 
$\int_{0}^{R} r^{n-1}\left(\int_{S^{n-1}} d \mathbf{S}\right) d r=\int_{S^{n-1}}\left(\int_{0}^{R} r^{n-1} d r\right) d \mathbf{S}=\frac{R^{n}\left|S^{n-1}\right|}{n}$, where the polar coordinates in $\mathbf{R}^{n}$ are used, and $|\mathbf{x}|$ denotes the Euclidean norm of the vector $\mathbf{x} \in \mathbf{R}^{n}$. Consequently, the volume of the unit ball $B(1)$ in $\mathbf{R}^{n}$ is

$$
|B(1)|=\frac{\left|S^{n-1}\right|}{n}, \quad \text { and } \quad|B(R)|=R^{n}|B(1)| .
$$

The basic result that will be followed here is due to M. Christ and L. Grafakos. In their paper [1] they proved the next direct generalization of (1) to the balls in $\mathbf{R}^{n}$ :

Theorem 2. Let $f \in L^{p}\left(\mathbf{R}^{n}\right)$ be a non-negative function, where $p>1$. The following inequality holds:

$$
\int_{\mathbf{R}^{n}}\left(\frac{1}{|B(|\mathbf{x}|)|} \int_{B(|\mathbf{x}|)} f(\mathbf{y}) d \mathbf{y}\right)^{p} d \mathbf{x} \leq\left(\frac{p}{p-1}\right)^{p} \int_{\mathbf{R}^{n}} f^{p}(\mathbf{y}) d \mathbf{y},
$$

and the constant $\left(\frac{p}{p-1}\right)^{p}$ is the best possible.

Inequality (3) was improved by P. Drábek, H. P. Heinig and A. Kufner in [4, where an analogue of (ID) is given. We state that result by

Theorem 3. If $p>1, k>1$ are real numbers, and $f$ is a non-negative function defined on $\mathbf{R}^{n}$ such that $|B(|\mathbf{x}|)|^{1-\frac{k}{p}} f \in L^{p}\left(\mathbf{R}^{n}\right)$, then

$$
\int_{\mathbf{R}^{n}}|B(|\mathbf{x}|)|^{-k}\left(\int_{B(|\mathbf{x}|)} f(\mathbf{y}) d \mathbf{y}\right)^{p} d \mathbf{x} \leq\left(\frac{p}{k-1}\right)^{p} \int_{\mathbf{R}^{n}}|B(|\mathbf{x}|)|^{p-k} f^{p}(\mathbf{x}) d \mathbf{x} .
$$

The constant $\left(\frac{p}{k-1}\right)^{p}$ is the best possible.

We also need to consider the one-dimensional weighted exponential inequalities, given in

Theorem 4. Let $\alpha$ and $\gamma$ be real numbers and $f$ be a positive function such that the function $t^{\alpha-1} \log f$ is locally integrable in $[0, \infty\rangle$. Then the inequalities

$$
\int_{0}^{\infty} x^{\gamma-1} \exp \left\{\frac{\alpha}{x^{\alpha}} \int_{0}^{x} t^{\alpha-1} \log f(t) d t\right\} d x \leq e^{\frac{\gamma}{\alpha}} \int_{0}^{\infty} x^{\gamma-1} f(x) d x
$$

for $\alpha>0$, and

$$
\int_{0}^{\infty} x^{\gamma-1} \exp \left\{-\frac{\alpha}{x^{\alpha}} \int_{x}^{\infty} t^{\alpha-1} \log f(t) d t\right\} d x \leq e^{\frac{\gamma}{\alpha}} \int_{0}^{\infty} x^{\gamma-1} f(x) d x
$$

for $\alpha<0$, hold. The constant $e^{\frac{\gamma}{\alpha}}$ is the best possible.

Originally, inequality (5) was discovered by V. Levin in his unnoticed paper [6], written in Russian, and then rediscovered by J. A. Cochran and C.-S. Lee in [2] (see also 8]). Because of this, inequality (5) will be called the Levin-Cochran-Lee inequality. Its companion result (6) was proved by E. R. Love in [7], and recently reproved by G.-S. Yang and Y.-J. Lin in [9].

In [4, weighted exponential inequalities of type (5) and (6), related to the balls in $\mathbf{R}^{n}$, were also examined, but very generally, without fixing any constant for the case of some particular radial weights.

In this paper we give another approach to inequalities (3) and (4) and derive their companion results. Also, the extensions of inequalities (5) and (6) to $n$-dimensional 
balls will be presented, including the proofs that the obtained constants are the best possible.

A technique that will be used here is based on introducing two types of multidimensional integral means of arbitrary real order, with power weights, independently of all stated theorems, and on proving the corresponding mixed-means inequalities. All the desired results will be obtained in an elegant way as limit cases of related mixed-means inequalities.

The idea of introducing mixed-means, and then using them for deriving Hardy and Levin-Cochran-Lee type inequalities has already been applied to one-dimensional integrals, finite and infinite series (cf. [3]).

The analysis used in the proofs is mostly based on classical real analysis, on the well-known Minkowski and Jensen inequalities for integrals, and on the properties of integral means (cf. [5]).

In what follows, without further explanation, we assume that all the integrals exist on the respective domains of their definitions.

\section{INTEGRAL MEANS AND RELATED MIXED-MEANS INEQUALITIES}

We start with the basic inequality.

Theorem 5. Let $r, s, R, \alpha, \gamma \in \mathbf{R}$ be such that $r<s, r, s \neq 0, R>0$. If $f$ is a non-negative function on $\mathbf{R}^{n}$ ( $f$ positive in the case $r<0$ ), then

$$
\begin{gathered}
\left\{\frac{1}{|B(R)|^{\alpha}} \int_{B(R)}|B(|\mathbf{x}|)|^{\alpha-1}\left[\frac{1}{|B(|\mathbf{x}|)|^{\gamma}} \int_{B(|\mathbf{x}|)}|B(|\mathbf{y}|)|^{\gamma-1} f^{s}(\mathbf{y}) d \mathbf{y}\right]^{\frac{r}{s}} d \mathbf{x}\right\}^{\frac{1}{r}} \\
(7) \geq\left\{\frac{1}{|B(R)|^{\gamma}} \int_{B(R)}|B(|\mathbf{x}|)|^{\gamma-1}\left[\frac{1}{|B(|\mathbf{x}|)|^{\alpha}} \int_{B(|\mathbf{x}|)}|B(|\mathbf{y}|)|^{\alpha-1} f^{r}(\mathbf{y}) d \mathbf{y}\right]^{\frac{s}{r}} d \mathbf{x}\right\}^{\frac{1}{s}}
\end{gathered}
$$

and

$$
\begin{aligned}
& \left\{\frac{1}{|B(R)|^{\alpha}} \int_{\mathbf{R}^{n} \backslash B(R)}|B(|\mathbf{x}|)|^{\alpha-1}\left[\frac{1}{|B(|\mathbf{x}|)|^{\gamma}} \int_{\mathbf{R}^{n} \backslash B(|\mathbf{x}|)}|B(|\mathbf{y}|)|^{\gamma-1} f^{s}(\mathbf{y}) d \mathbf{y}\right]^{\frac{r}{s}} d \mathbf{x}\right\}^{\frac{1}{r}} \\
& \geq\left\{\frac{1}{|B(R)|^{\gamma}} \int_{\mathbf{R}^{n} \backslash B(R)}|B(|\mathbf{x}|)|^{\gamma-1}\left[\frac{1}{|B(|\mathbf{x}|)|^{\alpha}} \int_{\mathbf{R}^{n} \backslash B(|\mathbf{x}|)}|B(|\mathbf{y}|)|^{\alpha-1} f^{r}(\mathbf{y}) d \mathbf{y}\right]^{\frac{s}{r}} d \mathbf{x}\right\}^{\frac{1}{s}} .
\end{aligned}
$$

Equality holds if and only if $f$ is of the form $f(\mathbf{x})=\phi(|\mathbf{x}|), \mathbf{x} \in \mathbf{R}^{n}$, where $\phi$ is such that $\phi(u v)=\phi_{1}(u) \phi_{2}(v)$, for $0<u<R, 0<v<1$ in (7), or $u>R, v>1$ in (8).

Proof. Let us prove (7) first. Using (2) and the change $\mathbf{z}=\frac{1}{\mid \mathbf{x}} \mathbf{y}$ of the independent variable in the inner integral, the right-hand side of (7) becomes

$$
\left\{\frac{1}{|B(R)|^{\gamma}} \int_{B(R)}|B(|\mathbf{x}|)|^{\gamma-1}\left[\frac{1}{|B(1)|} \int_{B(1)}|\mathbf{z}|^{n(\alpha-1)} f^{r}(|\mathbf{x}| \mathbf{z}) d \mathbf{z}\right]^{\frac{s}{r}} d \mathbf{x}\right\}^{\frac{1}{s}} .
$$


Further, it is equal to

$$
\left\{\frac{1}{|B(R)|^{\gamma}} \int_{B(R)}|B(|\mathbf{x}|)|^{\gamma-1}\left[\frac{1}{\left|S^{n-1}\right|} \int_{S^{n-1}}\left(n \int_{0}^{1} v^{n \alpha-1} f^{r}(|\mathbf{x}| v \mathbf{S}) d v\right) d \mathbf{S}\right]^{\frac{s}{r}} d \mathbf{x}\right\}^{\frac{1}{s}},
$$

where polar coordinates and (2) are used. By applying Jensen's inequality to the term in the square brackets in (9) and to the function $t \mapsto t^{\frac{s}{r}}$, we have that (9) is less than or equal to

$$
\begin{aligned}
& \left\{\frac{1}{|B(R)|^{\gamma}\left|S^{n-1}\right|} \int_{B(R)}|B(|\mathbf{x}|)|^{\gamma-1} \int_{S^{n-1}}\left[n \int_{0}^{1} v^{n \alpha-1} f^{r}(|\mathbf{x}| v \mathbf{S}) d v\right]^{\frac{s}{r}} d \mathbf{S} d \mathbf{x}\right\}^{\frac{1}{s}} \\
& =\left\{\frac{1}{|B(R)|^{\gamma}\left|S^{n-1}\right|} \int_{S^{n-1}} \int_{0}^{R} u^{n-1}|B(u)|^{\gamma-1}\right. \\
& \left.\int_{S^{n-1}}\left[n \int_{0}^{1} v^{n \alpha-1} f^{r}(u v \mathbf{S}) d v\right]^{\frac{s}{r}} d \mathbf{S} d u d \mathbf{T}\right\}^{\frac{1}{s}} \\
& =\left\{\frac { 1 } { | B ( R ) | ^ { \gamma } } \int _ { 0 } ^ { R } u ^ { n - 1 } | B ( u ) | ^ { \gamma - 1 } \int _ { S ^ { n - 1 } } \left[\frac{n}{\left|S^{n-1}\right|}\right.\right. \\
& \left.\left.\int_{S^{n-1}} \int_{0}^{1} v^{n \alpha-1} f^{r}(u v \mathbf{S}) d v d \mathbf{T}\right]^{\frac{s}{r}} d \mathbf{S} d u\right\}^{\frac{1}{s}} \\
& =\left\{\frac{1}{|B(R)|^{\gamma}} \int_{B(R)}|B(|\mathbf{x}|)|^{\gamma-1}\left[\frac{1}{|B(1)|^{\alpha}} \int_{B(1)}|B(|\mathbf{z}|)|^{\alpha-1} f^{r}(|\mathbf{z}| \mathbf{x}) d \mathbf{z}\right]^{\frac{s}{r}} d \mathbf{x}\right\}^{\frac{1}{s}} .
\end{aligned}
$$

The sequence of equalities that yields (10) is obtained by transforming the first integral to polar coordinates and then back to Cartesian coordinates. Using Minkowski's integral inequality, (10) is less than or equal to

$$
\left\{\frac{1}{|B(1)|^{\alpha}} \int_{B(1)}|B(|\mathbf{z}|)|^{\alpha-1}\left[\frac{1}{|B(R)|^{\gamma}} \int_{B(R)}|B(|\mathbf{x}|)|^{\gamma-1} f^{s}(|\mathbf{z}| \mathbf{x}) d \mathbf{x}\right]^{\frac{r}{s}} d \mathbf{z}\right\}^{\frac{1}{r}}
$$

By substituting back $\mathbf{y}=|\mathbf{z}| \mathbf{x}$, (11) is equal to

$$
\begin{aligned}
& \left\{\frac{1}{|B(1)|^{\alpha}} \int_{B(1)}|B(|\mathbf{z}|)|^{\alpha-1}\left[\frac{1}{|B(|\mathbf{z}| R)|^{\gamma}} \int_{B(|\mathbf{z}| R)}|B(|\mathbf{y}|)|^{\gamma-1} f^{s}(\mathbf{y}) d \mathbf{y}\right]^{\frac{r}{s}} d \mathbf{z}\right\}^{\frac{1}{r}} \\
& =\left\{\frac{1}{|B(R)|^{\alpha}} \int_{B(R)}|B(|\mathbf{x}|)|^{\alpha-1}\left[\frac{1}{|B(|\mathbf{x}|)|^{\gamma}} \int_{B(|\mathbf{x}|)}|B(|\mathbf{y}|)|^{\gamma-1} f^{s}(\mathbf{y}) d \mathbf{y}\right]^{\frac{r}{s}} d \mathbf{x}\right\}^{\frac{1}{r}}
\end{aligned}
$$

that is, the left-hand side of (17). The last equality is due to the substitution $\mathbf{x}=R \mathbf{z}$. 
Now, (8) can be easily proved if (7) is written for $-\alpha,-\gamma, \frac{1}{R}, g(\mathbf{x})=f\left(\frac{\mathbf{x}}{|\mathbf{x}|^{2}}\right)$, instead of $\alpha, \gamma, R, f(\mathbf{x})$, and the substitutions $\mathbf{z}=\frac{\mathbf{y}}{|\mathbf{y}|^{2}}$ in the inner integrals, and then $\mathbf{w}=\frac{\mathbf{x}}{|\mathbf{x}|^{2}}$ in the first integrals, on the both sides of the inequality, are used.

Finally, let us give the necessary and sufficient conditions for achieving equalities in (7) and (8). It is obvious that in (7) the equality sign will occur if function $f$ fulfills the conditions from the statement of Theorem 5 Vice-versa, suppose that the equality in 7 holds. In this case, there must be the equality in Minkowski's inequality, so $f(|\mathbf{z}| \mathbf{x})=f_{1}(\mathbf{x}) \phi_{2}(|\mathbf{z}|), \mathbf{x} \in B(R), \mathbf{z} \in B(1)$. Further, equality must also hold in Jensen's inequality. Hence, $f_{1}(\mathbf{x})=\phi_{1}(|\mathbf{x}|)$, or $f(|\mathbf{z}| \mathbf{x})=\phi_{1}(|\mathbf{x}|) \phi_{2}(|\mathbf{z}|)$, $\mathbf{x} \in B(R), \mathbf{z} \in B(1)$. Especially, $f(\mathbf{x})=\phi_{1}(|\mathbf{x}|) \phi_{2}(1), \mathbf{x} \in B(R)$, so $f$ is radial, i.e. $f(\mathbf{x})=\phi(|\mathbf{x}|)$, and

$$
\phi_{1}(|\mathbf{x}|) \phi_{2}(|\mathbf{z}|)=f(|\mathbf{z}| \mathbf{x})=\phi(|\mathbf{x}||\mathbf{z}|), \quad \mathbf{x} \in B(R), \mathbf{z} \in B(1),
$$

so the conditions stated in Theorem 5 are fulfilled. The conditions for equality in (8) can be derived by the same arguments.

We continue by introducing integral power means and their natural companion means. Let $R \in \mathbf{R}, R>0$, and let $f$ be a non-negative function on $\mathbf{R}^{n}$. For $r, \alpha \in \mathbf{R}, r \neq 0, \alpha>0$, as in [5], we define the integral mean of order $r$, with the power weight, of $f, M_{r}(f ; R, \alpha)$, by

$$
M_{r}(f ; R, \alpha)=\left[\frac{\alpha}{|B(R)|^{\alpha}} \int_{B(R)}|B(|\mathbf{x}|)|^{\alpha-1} f^{r}(\mathbf{x}) d \mathbf{x}\right]^{\frac{1}{r}},
$$

with the convention that $M_{r}(f ; R, \alpha)=0$ if $r<0$ and $f$ vanishes on a subset of $B(R)$ of positive measure.

On the other hand, if $\alpha<0$, we define the companion mean of order $r$ of $f$, $M_{r}^{*}(f ; R, \alpha)$, by

$$
M_{r}^{*}(f ; R, \alpha)=\left[-\frac{\alpha}{|B(R)|^{\alpha}} \int_{\mathbf{R}^{n} \backslash B(R)}|B(|\mathbf{x}|)|^{\alpha-1} f^{r}(\mathbf{x}) d \mathbf{x}\right]^{\frac{1}{r}},
$$

where we set $M_{r}^{*}(f ; R, \alpha)=0$ if $f(\mathbf{x})=0$ on a subset of $\mathbf{R}^{n} \backslash B(R)$ of positive measure.

In particular, for a positive function $f$, if $\alpha>0$, let $G(f ; R, \alpha)$ denote its geometric mean,

$$
G(f ; R, \alpha)=M_{0}(f ; R, \alpha)=\exp \left(\frac{\alpha}{|B(R)|^{\alpha}} \int_{B(R)}|B(|\mathbf{x}|)|^{\alpha-1} \log f(\mathbf{x}) d \mathbf{x}\right),
$$

while for $\alpha<0$ let $G^{*}(f ; R, \alpha)$ be the companion geometric mean of $f$, given by

$$
G^{*}(f ; R, \alpha)=M_{0}^{*}(f ; R, \alpha)=\exp \left(-\frac{\alpha}{|B(R)|^{\alpha}} \int_{\mathbf{R}^{n} \backslash B(R)}|B(|\mathbf{x}|)|^{\alpha-1} \log f(\mathbf{x}) d \mathbf{x}\right) .
$$

The means have the further properties (cf. [5]):

$$
M_{r}(f ; R, \alpha) \leq M_{s}(f ; R, \alpha), M_{r}^{*}(f ; R, \alpha) \leq M_{s}^{*}(f ; R, \alpha),
$$

for $r<s$, and

$$
\lim _{r \rightarrow 0} M_{r}(f ; R, \alpha)=G(f ; R, \alpha), \lim _{r \rightarrow 0} M_{r}^{*}(f ; R, \alpha)=G^{*}(f ; R, \alpha) .
$$


Note that Theorem 5 avoided cases where $r=0$ or $s=0$. Results related to these situations are given in the next theorem.

Theorem 6. Let $f$ be a positive function on $\mathbf{R}^{n}$, and let $s, R, \alpha, \gamma \in \mathbf{R}$ be such that $s, R>0, \alpha \neq 0$. If $\alpha>0$, then

$$
\begin{aligned}
& \left\{\frac{1}{|B(R)|^{\gamma}} \int_{B(R)}|B(|\mathbf{x}|)|^{\gamma-1}\left[\exp \left(\frac{\alpha}{|B(|\mathbf{x}|)|^{\alpha}} \int_{B(|\mathbf{x}|)}|B(|\mathbf{y}|)|^{\alpha-1} \log f(\mathbf{y}) d \mathbf{y}\right)\right]^{s} d \mathbf{x}\right\}^{\frac{1}{s}} \\
\leq & \exp \left[\frac{\alpha}{|B(R)|^{\alpha}} \int_{B(R)}|B(|\mathbf{x}|)|^{\alpha-1} \log \left(\frac{1}{|B(|\mathbf{x}|)|^{\gamma}} \int_{B(|\mathbf{x}|)}|B(|\mathbf{y}|)|^{\gamma-1} f^{s}(\mathbf{y}) d \mathbf{y}\right)^{\frac{1}{s}} d \mathbf{x}\right],
\end{aligned}
$$

and if $\alpha<0$, then

$$
\begin{aligned}
& \left\{\frac{1}{|B(R)|^{\gamma}} \int_{\mathbf{R}^{n} \backslash B(R)}|B(|\mathbf{x}|)|^{\gamma-1}\left[\exp \left(-\frac{\alpha}{|B(|\mathbf{x}|)|^{\alpha}} \int_{\mathbf{R}^{n} \backslash B(|\mathbf{x}|)}|B(|\mathbf{y}|)|^{\alpha-1} \log f(\mathbf{y}) d \mathbf{y}\right)\right]^{s} d \mathbf{x}\right\} \\
\leq & \exp \left[-\frac{\alpha}{|B(R)|^{\alpha}} \int_{\mathbf{R}^{n} \backslash B(R)}|B(|\mathbf{x}|)|^{\alpha-1} \log \left(\frac{1}{|B(|\mathbf{x}|)|^{\gamma}} \int_{\mathbf{R}^{n} \backslash B(|\mathbf{x}|)}|B(|\mathbf{y}|)|^{\gamma-1} f^{s}(\mathbf{y}) d \mathbf{y}\right)^{\frac{1}{s}} d \mathbf{x}\right] .
\end{aligned}
$$

Proof. Define $h(\mathbf{x})=\left[\frac{1}{|B(|\mathbf{x}|)|^{\gamma}} \int_{B(|\mathbf{x}|)}|B(|\mathbf{y}|)|^{\gamma-1} f^{s}(\mathbf{y}) d \mathbf{y}\right]^{\frac{1}{s}}$, for $\mathbf{x} \in B(R)$. For any $0<r<s$ and $\alpha>0$, applying (7) to the function $\alpha^{\frac{1}{r}} f$ instead of $f$ and using (12), we obtain that

$$
\left\{\frac{1}{|B(R)|^{\gamma}} \int_{B(R)}|B(|\mathbf{x}|)|^{\gamma-1}\left[M_{r}(f ;|\mathbf{x}|, \alpha)\right]^{s} d \mathbf{x}\right\}^{\frac{1}{s}} \leq M_{r}(h ; R, \alpha) .
$$

Since $M_{r}(f ;|\mathbf{x}|, \alpha) \geq 0$, and, by relations (16) and (17), it converges monotonically to $G(f ;|\mathbf{x}|, \alpha)$, as $r$ decreases to 0 , Lebesgue's monotone convergence theorem implies that the limit of the left-hand side of (20), as $r \backslash 0$, is equal to

$$
\left\{\frac{1}{|B(R)|^{\gamma}} \int_{B(R)}|B(|\mathbf{x}|)|^{\gamma-1}[G(f ;|\mathbf{x}|, \alpha)]^{s} d \mathbf{x}\right\}^{\frac{1}{s}} .
$$

Moreover, (17) also implies that $\lim _{r \backslash 0} M_{r}(h ; R, \alpha)=G(h ; R, \alpha)$, so (18) holds by taking $\lim _{r \backslash 0}$ of (20).

Inequality (19) can be proved by the same tools, if we rewrite (8) for $0<r<s$, $\alpha<0$, and $(-\alpha)^{\frac{1}{r}} f$ instead of $f$.

We conclude this section with the main result, the mixed $(r, s)$-means inequalities for means $M$ and their companion means, $M^{*}$.

Theorem 7. Suppose $f$ is a positive function defined on $\mathbf{R}^{n}$. If $r, s, R, \alpha$ and $\gamma$ are real numbers such that $R>0$ and $r<s$, then

(i) $M_{r}\left(M_{s}(f ;|\mathbf{x}|, \gamma) ; R, \alpha\right) \geq M_{s}\left(M_{r}(f ;|\mathbf{x}|, \alpha) ; R, \gamma\right)$, for $\alpha, \gamma>0$;

(ii) $M_{r}^{*}\left(M_{s}^{*}(f ;|\mathbf{x}|, \gamma) ; R, \alpha\right) \geq M_{s}^{*}\left(M_{r}^{*}(f ;|\mathbf{x}|, \alpha) ; R, \gamma\right)$, for $\alpha, \gamma<0$. 
Proof. This theorem is direct consequence of Theorem 5 applied to the function $\alpha^{\frac{1}{r}} \gamma^{\frac{1}{s}} f$ in (i), and to the $(-\alpha)^{\frac{1}{r}}(-\gamma)^{\frac{1}{s}} f$ in (ii). The analysis is the same as in the proof of Theorem 6 .

\section{HARdy TYPe INEQUALITIES}

The mixed means and related inequalities are of use in their application in proving different integral inequalities. For example, inequalities (7) and (8), established in the previous section, can be used as an approach to Hardy's inequality (4). Here we give the companion inequality to (4) and new proofs of both of them.

A direct generalization of Theorem 1 to multidimensional balls is given in

Theorem 8. Let $p>1$ and $k \neq 1$ be real numbers. If $f$ is a non-negative function on $\mathbf{R}^{n}$ such that $|B(|\mathbf{x}|)|^{1-\frac{k}{p}} f \in L^{p}\left(\mathbf{R}^{n}\right)$, and the function $F$ is defined on $\mathbf{R}^{n}$ by

$$
F(\mathbf{x})= \begin{cases}\int_{B(|\mathbf{x}|)} f(\mathbf{y}) d \mathbf{y}, & k>1, \\ \int_{\mathbf{R}^{n} \backslash B(|\mathbf{x}|)} f(\mathbf{y}) d \mathbf{y}, & k<1,\end{cases}
$$

then

$$
\int_{\mathbf{R}^{n}}|B(|\mathbf{x}|)|^{-k}[F(\mathbf{x})]^{p} d \mathbf{x} \leq\left(\frac{p}{|k-1|}\right)^{p} \int_{\mathbf{R}^{n}}|B(|\mathbf{x}|)|^{-k}[|B(|\mathbf{x}|)| f(\mathbf{x})]^{p} d \mathbf{x} .
$$

The constant $\left(\frac{p}{|k-1|}\right)^{p}$ is the best possible.

Proof. Let $r=1, s=p>1, \alpha=1, \gamma=p-k+1$ in Theorem [5, and let $R>0$ be arbitrary. Consider the case $k>1$ first. Using the given parameters, inequality (7) can be written in the form

$$
\begin{gathered}
\int_{B(R)}|B(|\mathbf{x}|)|^{-k}[F(\mathbf{x})]^{p} d \mathbf{x} \leq|B(R)|^{1-k}\left\{\int_{B(R)}|B(|\mathbf{x}|)|^{\frac{k-1}{p}-1}\right. \\
\left.\cdot\left[\int_{B(|\mathbf{x}|)}|B(|\mathbf{y}|)|^{-k}[|B(|\mathbf{y}|)| f(\mathbf{y})]^{p} d \mathbf{y}\right]^{\frac{1}{p}} d \mathbf{x}\right\}^{p} .
\end{gathered}
$$

Since $I_{R}=\int_{B(R)}|B(|\mathbf{y}|)|^{p-k}[f(\mathbf{y})]^{p} d \mathbf{y} \geq \int_{B(|\mathbf{x}|)}|B(|\mathbf{y}|)|^{p-k}[f(\mathbf{y})]^{p} d \mathbf{y}, \mathbf{x} \in B(R)$, the right-hand side of (22) is not greater than

$$
|B(R)|^{1-k}\left[\int_{B(R)}|B(|\mathbf{x}|)|^{\frac{k-1}{p}-1} d \mathbf{x}\right]^{p} \cdot I_{R}=\left(\frac{p}{k-1}\right)^{p} \cdot I_{R}
$$

where the relation $\int_{B(R)}|B(|\mathbf{x}|)|^{\frac{k-1}{p}-1} d \mathbf{x}=\frac{p}{k-1}|B(R)|^{\frac{k-1}{p}}$ is obtained using elementary calculus. Inequality (21) is now derived by taking $\lim _{R \rightarrow \infty}$.

For the case $k<1$, inequality (21) is proved analogously, but this time by starting from (8), then taking a similar estimate as in the previous case, and finally, by taking $\lim _{R \rightarrow 0}$. Observe that $\int_{\mathbf{R}^{n} \backslash B(R)}|B(|\mathbf{x}|)|^{\frac{k-1}{p}-1} d \mathbf{x}=\frac{p}{1-k}|B(R)|^{\frac{k-1}{p}}$.

The best constant for (21), the case $k>1$, is discussed in [1] and [4]. Inequalities (21) are mutually equivalent, since by writing one of them for $2-k$ and $|B(1)|^{\frac{2(1-k)}{p}}|\mathbf{x}|^{-2 n} f\left(\frac{\mathbf{x}}{|\mathbf{x}|^{2}}\right)$ instead of $k$ and $f$ and using the substitutions $\mathbf{z}=\frac{\mathbf{y}}{|\mathbf{y}|^{2}}$ and $\mathbf{w}=\frac{\mathbf{x}}{|\mathbf{x}|^{2}}$ on the left-hand side and $\mathbf{w}=\frac{\mathbf{x}}{|\mathbf{x}|^{2}}$ on the right-hand side we obtain 
the other inequality. So, the best constant for $k>1$ is obviously also the best constant for the case $k<1$ in (21).

Remark 1. Note that the introducing of arbitrary parameter $\alpha$ in (21) does not contribute to greater generality of Hardy's inequality, since the function $f$ can always be replaced with $|B(|\mathbf{x}|)|^{\alpha-1} f$.

\section{LEVIN-COChran-LEe TYPe INEQUALITIES}

By taking the limits of inequalities (18) and (19), we obtain multivariable versions of (5) and (6) related to balls.

Theorem 9. If $f$ is a positive function on $\mathbf{R}^{n}$, and $\alpha, \gamma \in \mathbf{R}$, then the inequalities

$$
\begin{gathered}
\int_{\mathbf{R}^{n}}|B(|\mathbf{x}|)|^{\gamma-1} \\
\exp \left(\frac{\alpha}{|B(|\mathbf{x}|)|^{\alpha}} \int_{B(|\mathbf{x}|)}|B(|\mathbf{y}|)|^{\alpha-1} \log f(\mathbf{y}) d \mathbf{y}\right) d \mathbf{x} \\
\leq e^{\frac{\gamma}{\alpha}} \int_{\mathbf{R}^{n}}|B(|\mathbf{x}|)|^{\gamma-1} f(\mathbf{x}) d \mathbf{x},
\end{gathered}
$$

for $\alpha>0$, and

$$
\begin{gathered}
\int_{\mathbf{R}^{n}}|B(|\mathbf{x}|)|^{\gamma-1} \exp \left(-\frac{\alpha}{|B(|\mathbf{x}|)|^{\alpha}} \int_{\mathbf{R}^{n} \backslash B(|\mathbf{x}|)}|B(|\mathbf{y}|)|^{\alpha-1} \log f(\mathbf{y}) d \mathbf{y}\right) d \mathbf{x} \\
\leq e^{\frac{\gamma}{\alpha}} \int_{\mathbf{R}^{n}}|B(|\mathbf{x}|)|^{\gamma-1} f(\mathbf{x}) d \mathbf{x},
\end{gathered}
$$

for $\alpha<0$, hold. The constant $e^{\frac{\gamma}{\alpha}}$ is the best possible.

Proof. Put $s=1$ in Theorem 6. Since

$$
J_{R}=\int_{B(R)}|B(|\mathbf{y}|)|^{\gamma-1} f(\mathbf{y}) d \mathbf{y} \geq \int_{B(|\mathbf{x}|)}|B(|\mathbf{y}|)|^{\gamma-1} f(\mathbf{y}) d \mathbf{y}, \quad \mathbf{x} \in B(R),
$$

and $\int_{B(R)}|B(|\mathbf{x}|)|^{\alpha-1} \log |B(|\mathbf{x}|)| d \mathbf{x}=\frac{|B(R)|^{\alpha}}{\alpha}\left(\log |B(R)|-\frac{1}{\alpha}\right), \alpha>0$, for arbitrary $\alpha, R>0$ from (18) we have

$$
\begin{aligned}
& \int_{B(R)}|B(|\mathbf{x}|)|^{\gamma-1} \exp \left(\frac{\alpha}{|B(|\mathbf{x}|)|^{\alpha}} \int_{B(|\mathbf{x}|)}|B(|\mathbf{y}|)|^{\alpha-1} \log f(\mathbf{y}) d \mathbf{y}\right) d \mathbf{x} \\
\leq & |B(R)|^{\gamma} \exp \left[\frac{\alpha}{|B(R)|^{\alpha}} \int_{B(R)}|B(|\mathbf{x}|)|^{\alpha-1} \log \left(\frac{1}{|B(|\mathbf{x}|)|^{\gamma}} \int_{B(|\mathbf{x}|)}|B(|\mathbf{y}|)|^{\gamma-1} f(\mathbf{y}) d \mathbf{y}\right) d \mathbf{x}\right] \\
\leq & \exp \left[\gamma \log |B(R)|+\frac{\alpha}{|B(R)|^{\alpha}}\left(\log J_{R} \cdot \int_{B(R)}|B(|\mathbf{x}|)|^{\alpha-1} d \mathbf{x}\right.\right. \\
& \left.\left.-\gamma \int_{B(R)}|B(|\mathbf{x}|)|^{\alpha-1} \log |B(|\mathbf{x}|)| d \mathbf{x}\right)\right]=e^{\frac{\gamma}{\alpha}} \cdot J_{R} .
\end{aligned}
$$

Inequality (23) follows by taking the $\lim _{R \rightarrow \infty}$. On the other hand, (24) is the consequence of (19), derived by the same technique as (23) from (18), except that we take the $\lim _{R \rightarrow 0}$. 
The proof that $e^{\frac{\gamma}{\alpha}}$ is the best possible constant for inequalities (23) and (24) follows. For any $\varepsilon>0$ and the function $f_{\varepsilon}$ defined by

$$
f_{\varepsilon}(\mathbf{x})= \begin{cases}\alpha e^{-\frac{\gamma}{\alpha}}|B(1)|^{-\gamma}|\mathbf{x}|^{-n(\gamma-\alpha \varepsilon)}, & \mathbf{x} \in B(1), \\ \alpha e^{-\frac{\gamma}{\alpha}}|B(1)|^{-\gamma}|\mathbf{x}|^{-n(\gamma+\alpha \varepsilon)}, & \mathbf{x} \in \mathbf{R}^{n} \backslash B(1),\end{cases}
$$

using the polar coordinates and the fact that $f_{\varepsilon}$ is radial, the left-hand side of (23) is equal to:

$$
\begin{aligned}
L= & \int_{B(1)}|B(|\mathbf{x}|)|^{\gamma-1} \exp \left(\frac{\alpha}{|B(|\mathbf{x}|)|^{\alpha}} \int_{B(|\mathbf{x}|)}|B(|\mathbf{y}|)|^{\alpha-1} \log f_{\varepsilon}(\mathbf{y}) d \mathbf{y}\right) d \mathbf{x} \\
& +\int_{\mathbf{R}^{n} \backslash B(1)}|B(|\mathbf{x}|)|^{\gamma-1} \exp \left[\frac { \alpha } { | B ( | \mathbf { x } | ) | ^ { \alpha } } \left(\int_{B(1)}|B(|\mathbf{y}|)|^{\alpha-1} \log f_{\varepsilon}(\mathbf{y}) d \mathbf{y}\right.\right. \\
& \left.\left.+\int_{B(|\mathbf{x}|) \backslash B(1)}|B(|\mathbf{y}|)|^{\alpha-1} \log f_{\varepsilon}(\mathbf{y}) d \mathbf{y}\right)\right] d \mathbf{x} \\
= & n \alpha\left(e^{-\varepsilon} \int_{0}^{1} u^{n \alpha \varepsilon-1} d u+e^{\varepsilon} \int_{1}^{\infty} u^{-n \alpha \varepsilon-1} e^{-\frac{2 \varepsilon}{u^{n \alpha}}} d u\right) \\
\geq & n \alpha e^{-\varepsilon}\left(\int_{0}^{1} u^{n \alpha \varepsilon-1} d u+\int_{1}^{\infty} u^{-n \alpha \varepsilon-1} d u\right)=\frac{2}{\varepsilon} \cdot e^{-\varepsilon},
\end{aligned}
$$

where the last row is obtained from the estimate $e^{-\frac{2 \varepsilon}{u^{n \alpha}}}>e^{-2 \varepsilon}, u>1$. A straightforward computation yields the right-hand side of (23):

$$
\begin{aligned}
R & =e^{\frac{\gamma}{\alpha}}\left(\int_{B(1)}|B(|\mathbf{x}|)|^{\gamma-1} f_{\varepsilon}(\mathbf{x}) d \mathbf{x}+\int_{\mathbf{R}^{n} \backslash B(1)}|B(|\mathbf{x}|)|^{\gamma-1} f_{\varepsilon}(\mathbf{x}) d \mathbf{x}\right) \\
& =n \alpha\left(\int_{0}^{1} u^{n \alpha \varepsilon-1} d u+\int_{1}^{\infty} u^{-n \alpha \varepsilon-1} d u\right)=\frac{2}{\varepsilon} .
\end{aligned}
$$

Since $1 \leq \frac{R}{L} \leq e^{\varepsilon} \rightarrow 1$, as $\varepsilon \rightarrow 0$, the desired result is proved. The proof that the constant $e^{\frac{\gamma}{a}}$ is the best possible for (24) is similar, if the function

$$
f_{\varepsilon}(\mathbf{x})= \begin{cases}\alpha e^{-\frac{\gamma}{\alpha}}|B(1)|^{-\gamma}|\mathbf{x}|^{-n(\gamma+\alpha \varepsilon)}, & \mathbf{x} \in B(1), \\ \alpha e^{-\frac{\gamma}{\alpha}}|B(1)|^{-\gamma}|\mathbf{x}|^{-n(\gamma-\alpha \varepsilon)}, & \mathbf{x} \in \mathbf{R}^{n} \backslash B(1),\end{cases}
$$

is considered.

\section{REFERENCES}

[1] M. Christ and L. Grafakos, Best constants for two nonconvolution inequalities, Proc. AMS 123, No. 6 (1995), 1687-1693. MR 95g:42031

[2] J. A. Cochran and C.-S. Lee, Inequalities related to Hardy's and Heinig's, Math. Proc. Cambridge Phil. Soc. 96 (1984), 1-7. MR 86g:26026

[3] A. Čižmešija and J. Pečarić, Mixed means and Hardy's inequality, Math. Inequal. Appl. 1, No. 4 (1998), 497-506. CMP 99:02

[4] P. Drábek, H. P. Heinig and A. Kufner, Higher dimensional Hardy inequality, Int. Ser. Num. Math. 123 (1997), 3-16. MR 98k:26026

[5] G. Hardy, J. E. Littlewood and G. Pólya, Inequalities, second edition, Cambridge University Press, Cambridge, 1967. MR 13:727e MR 89d:26016

[6] V. Levin, O neravenstvah III: Neravenstva, vypolnjaemie geometričeskim srednim neotricatel'noi funkcii, Math. Sbornik 4 ( 46) (1938), 325-331.

[7] E.R. Love, Inequalities related to those of Hardy and of Cochran and Lee, Math. Proc. Cambridge Phil. Soc. 99 (1986), 395-408. MR 87f:26021 
[8] D. S. Mitrinović, J. E. Pečarić and A. M. Fink, Inequalities involving functions and their integrals and derivatives, Kluwer Academic Publishers, 1991. MR 93m:26036

[9] G.-S. Yang, Y.-J. Lin, On companion inequalities related to Heinig's, Tamkang Journal of Math., 22 (1991), No. 4, 313-322. MR 93c:26021

Department of Mathematics, University of Zagreb, BijeničKacesta 30, 10000 Zagreb, CROATIA

E-mail address: cizmesij@math.hr

Faculty of Textile Technology, University of Zagreb, Pierottijeva 6, 10000 Zagreb, Croatia

E-mail address: pecaric@mahazu.hazu.hr

Faculty of Chemical Engineering and Technology, University of Zagreb, Marulićev trg 19, 10000 Zagreb, Croatia 